\title{
Tratamiento de los géneros periodísticos en la información de la prensa euromediterránea
}

\author{
Fernando SABÉS TURMO \\ Universidad Autónoma de Barcelona \\ fernando.sabes@uab.es \\ Ricardo CARNiEl Bugs \\ Universidad Autónoma de Barcelona \\ ricado.carniel@uab.es
}

\begin{abstract}
Resumen:
La información generada en el espacio euromediterráneo es de gran importancia en la actualidad y no únicamente por lo que se conoce como primavera árabe, sino que anteriormente ya se trabajaba en diferentes foros como el Proceso de Barcelona-Unión por el Mediterráneo, la Política Europea de Vecindad de la Comisión Europea y las recomendaciones del Informe del Grupo de Alto Nivel de la Alianza de Civilizaciones reflejadas en el Plan de Acción 2007-2009. Este artículo analiza las géneros periodísticos utilizado por 16 diarios en el tratamiento de la información relativa al espacio euromediterráneo: $E l$ Khabar y El Watan (Argelia), Il Corriere della Sera y La Repubblica (Italia), ABC y El País (España), The Guardian y The Times (Gran Bretaña), Al Ahram y Al Dostur (Egipto), Al Massae y As Sabah (Marruecos), Le Figaro y Le Monde (Francia) y Al Thawra y Al Watan (Siria). En total se han estudiado 1.254 unidades de análisis recogidas entre el 9 y el 22 de marzo de 2009 poniendo de manifiesto que la noticia es el género informativo mayoritario y evidenciando una falta de innovación en la mayoría de las cabeceras estudiadas.
\end{abstract}

Palabras clave: Géneros periodísticos; Información; Euromediterráneo; Prensa

\section{Treatment of journalistic genres in the information of Euro-Mediterranean press}

\begin{abstract}
:
The information generated in the Euro-Mediterranean area has currently great importance, not only due to the events known as the Arab Spring. Previously, many initiatives were already underway in different forums such as the Barcelona Process: Union for the Mediterranean, the European Neighbourhood Policy of the European Commission, and the recommendations of the Report of the High Level Group of the Alliance of Civilizations reflected in the 2007-2009 Action Plan. This article analyzes the journalistic genres used by 16 newspapers in the treatment of the information related to the Euro-Mediterranean area: El Khabar and El Watan (Algeria), Il Corriere della Sera and La Repubblica (Italy), ABC and El Pais (Spain), The Guardian and The Times (UK), Al Ahram and Al Dostur (Egypt), Al Massae and As Sabah (Morocco), Le Figaro and Le Monde (France) and Al Thawra and Al Watan (Syria). In total, we examined 1.254 units of analysis which are collected between 9 and 22 March 2009. The results show that news is the major journalistic genre, indicating a lack of innovation in most of the analyzed newspapers.
\end{abstract}

Key words: Journalistic genres; Information; Mediterranean; Newspapers 


\section{Referencia normalizada:}

Sabés Turmo, F. y Carniel Bugs, R. (2013) Tratamiento de los geéneros periodísticos en la información de la prensa euromediterranea. Historia y Comunicación Social, Vol. 18. No Especial Octubre. Pags. $15-32$

Sumario: 1. Introducción. 2. Metodología y muestra. 2.1. Metodología. 2.2. Muestra seleccionada. 3. ¿Qué entendemos por géneros periodísticos? 4. Análisis de resultados. 5. Conclusiones. 6. Bibliografía

\section{Introducción}

El espacio euromediterráneo ha sido objeto de estudio a través de diferentes proyectos de investigación impulsados desde el Laboratori de Prospectiva i Recerca en Comunicació, Cultura i Cooperació (LAPREC) ${ }^{1}$ de la Universidad Autónoma de Barcelona, haciendo especial hincapié en el análisis de la información que en esa área se genera. Este interés por esta zona va mucho más allá de los últimos acontecimientos conocidos como Primavera Árabe, con sus derivadas posteriores. De hecho, los estudios que se han ido realizando parten de los objetivos del Proceso de Barcelona-Unión por el Mediterráneo ${ }^{2}$, la Política Europea de Vecindad de la Comisión Europea $^{3}$ y las recomendaciones del Informe del Grupo de Alto Nivel de la Alianza de Civilizaciones contenidas en el Plan de Acción 2007-2009 haciendo referencia a los aspectos vinculados a la comunicación.

Este artículo pretende analizar la utilización de los géneros periodísticos por los principales diarios de la región euromediterránea con el objetivo de ver si se produce un tratamiento diverso entre unos medios y otros a ambos lados del Mediterráneo. Para este estudio únicamente se abordarán los géneros informativos por los que los de opinión quedarán excluidos. Lo que se pretende es resumir como los diarios escogidos en la muestra de análisis realizan las coberturas informativas sobre determinados temas que afectan a la región y de acuerdo a los documentos e informes previamente citados. La pretendida comparación entre las cabeceras nos ofrecerá también la percepción que en los diferentes países se tiene de las noticias e informaciones sobre la región euromediterránea.

Los resultados que se presentan en este artículo corresponden al proyecto de investigación dirigido por la Dra. Teresa Velázquez García-Talavera titulado "La construcción social del espacio euromediterráneo en los medios de comunicación. La

1 http://grupsderecerca.uab.cat/laprec/es [17-10-2013]

$2 \mathrm{http} / /$ www.gencat.cat/relacions_exteriors/bcn10/cas/pdf/declaracio.pdf y http://www. ufmsecretariat.org/en/wp-content/uploads/2010/11/ufm_paris_declaration1.pdf [17-102013]

3 http://ec.europa.eu/world/enp/pdf/country/enpi_euromed_rsp_summary_es.pdf [17-102013]

$4 \mathrm{http}: / /$ www.unaoc.org/docs/AoC_HLG_REPORT_SP.pdf y http://www.unaoc.org/wp-content/uploads/071010-Implementation-Plan-1_2007̄-2009.pdf [17-10-2013] 
información en prensa y televisión" (CSO2008-01579) ${ }^{5}$, financiado por el Ministerio español de Ciencia e Innovación (2009-2012), que estudia la cobertura informativa de 16 diarios en su versión impresa de la zona mediterránea, tanto de Europa, como de África y Oriente Medio.

Esta investigación tiene su continuidad en la actualidad con un nuevo proyecto de investigación denominado "La construcción social del espacio intermediterráneo y sus correlaciones en la agenda temática de los medios de comunicación. La información en prensa y televisión" también financiado a través del Plan Nacional de investigación (CSO2012-35955) ${ }^{6}$.

\section{Metodología y muestra}

\subsection{Metodología}

El proyecto de investigación se ha desarrollado bajo una metodología de corte documental, a través inicialmente de dos tipos de fuentes, las estadísticas y las de audiencia de medios de comunicación, con el fin de conseguir datos referentes a las cabeceras analizadas. Aunque en este artículo se hace referencia únicamente a la prensa escrita, sí hay que señalar que el proyecto de investigación recoge además una muestra de los informativos de televisión de las principales cadenas de la región euromediterránea.

De todas maneras, hay que señalar que el análisis de contenido realizado se plantea desde la teoría de la construcción de la realidad social y en concreto en la manera en la que los medios de comunicación generan esa construcción: el contexto social

5 Los resultados contenidos en este artículo corresponden a la investigación denominada "La construcción social del espacio euro mediterráneo en los medios de comunicación. La información en prensa y televisión" (CSO2008-01579/SOCI), financiada por el Ministerio español de Ciencia e innovación (2009-2011). Investigadores por orden alfabético: Hassan Abbas, María Luz Barbeito, Tayeb Boutbouqalt, Ricardo Carniel Bugs, Carmina Crusafon, Olga Del Río, María Pilar Diezhandino Nieto, Natalia Fernández Díaz, Elisabet García Altadill, Juan Antonio García Galindo, Abdelouhad El Imrani, Francisco Martín, Carmen Martínez Romero, Lucía Molina, Nieves Ortega, José María Perceval, José Manuel Pérez Tornero, Fernando Sabés, Susana Tovías, Sahar Taalat. Teresa Velázquez (Investigadora Principal). Doctorandas adscritas al proyecto: Cristina Marques y Valentina Saini.

6 "La construcción social del espacio intermediterráneo y sus correlaciones en la agenda temática de los medios de comunicación. La información en prensa y televisión" (CSO2012-35955) es un proyecto financiado por el Ministerio español de Economía y Competitividad (2013-2015). Investigadores por orden alfabético: Hesham Abu-Sharar, María Luz Barbeito, Ricardo Carniel Bugs, Carmina Crusafon, Olga Del Río, Lucrecia Escudero Chauvel, Natalia Fernández Díaz, Elisabet García Altadill, Juan Antonio García Galindo, Víctor Luís Gutiérrez Castillo, Nouredinne Harrami, Carmen Martínez Romero, Patria Román-Velázquez, Fernando Sabés, Susana Tovías, José Juan Verón Lassa y Teresa Velázquez (Investigadora Principal). Doctorandos adscritos al proyecto: Estibaliz Ortega Miranda, Hicham El Bakhouti y Valentina Saini. Investigadores colaboradores del proyecto (pendientes de incorporación administrativa): Zhou Bouzidi y Carolina Cerdá-Guzmán. 
condiciona la realidad y el conocimiento establecido (Berger y Luckmann, 1976). Así, se puede afirmar que estamos en una tendencia fenomenológica-interpretativa desde el punto de vista teórico.

"Suele haber consenso en torno a la consideración de que la Sociología Fenomenológica se inserta en el campo de las llamadas Sociologías Interpretativas, junto a corrientes como el Interaccionismo Simbólico, las Sociologías de la Vida Cotidiana e incluso las Sociologías de la Situación" (Rizo, 2009: 25).

Por su parte, Velázquez (1992:22) asegura que:

"si lo medios de comunicación de masas contribuyen a la construcción de la realidad y la presentan en el espacio de la comunicación pública, los destinatarios de ese discurso también perciben y construyen realidad, bien la presentada por los medios, bien la individual según su conocimiento del mundo, valores, creencias, opiniones...".

Con todo este trabajo previo se llevó a cabo una selección de las cabeceras que iban a ser objeto de análisis, a través de una metodología de corte cuantitativa que se basaba en el análisis de contenido aplicado exclusivamente a las informaciones que aparecían relativas a la construcción social del espacio euromediterráneo de la comunicación. Para ello, se diseñó una ficha de estudio muy amplia que previamente fue probada a través de un pretest que dio validez al trabajo realizado hasta el momento.

Pero en el proyecto de investigación se fue más allá, con una tercera fase dentro de la metodología, en este caso cualitativa, realizando un análisis semiótico y del discurso que se aplicó a la muestra escogida, puesto que de lo que se trataba era analizar el "cómo se dice lo que se dice" para abordar el contenido latente del mensaje, su significado, de manera que se encuentren las claves para su interpretación (Velázquez, 2011: 237).

En el artículo que presentamos nos ceñiremos a la segunda de las fases de la metodología expuesta, es decir, al análisis de contenido desde la perspectiva cuantitativa de las informaciones aparecidas en las cabeceras estudiadas y que hacen referencia a los contenidos del espacio euromediterráneo. Holsti (1968) señala que el análisis de contenido es un procedimiento que permite analizar y cuantificar los materiales de la comunicación y puede estudiarse con detalle y profundidad el contenido de cualquier comunicación. "Técnica para realizar inferencias mediante la identificación sistemática y objetiva de características específicas de mensajes" (Holsti, 1968: 601).

Berelson describe el análisis de contenido como la "técnica de investigación para la descripción objetiva, sistemática y cuantitativa del contenido manifiesto de las comunicaciones, con el fin de interpretarlas" (Berelson, 1952: 18).

Bardín (1986: 32) señala que:

“el análisis de contenido es un conjunto de técnicas de análisis de comunicaciones que tiende a obtener indicadores (cuantitativos o no) por procedimientos sistemáticos y objetivos de descripción del contenido de los mensajes, permitiendo la inferen- 
cia de conocimientos relativos a las condiciones de producción/recepción (variables inferidas) de estos mensajes".

Por su parte, Krippendorff (1990:28), define el análisis de contenido como "la técnica destinada a formular, a partir de ciertos datos, inferencias reproducibles y válidas que puedan aplicarse a un contexto".

El principal objetivo que nos marcamos con este trabajo es comprobar el comportamiento de las diferentes cabeceras escogidas en la muestra en el uso de los géneros periodísticos informativos en el momento en el que abordan contenidos relativos al espacio euromediterráneo.

\subsection{Muestra seleccionada}

La muestra seleccionada corresponde al análisis de 16 diarios. El periodo de recolección de datos fue de dos semanas, en concreto del 9 al 22 de marzo de 2009. En total fueron 1.254 unidades de análisis, prácticamente repartidas al $50 \%$ entre ambas semanas, 653 la primera y 601 la segunda. Las cabeceras escogidas fueron: Al Ahram (Egipto), Al Dostur (Egipto), Al Massae (Marruecos), Al Thawra (Siria), Al Watan-Siria (Siria), As Sabah (Marruecos), El Khabar (Argelia), ABC (España), El País (España), El Watan (Argelia), Il Corriere della Sera (Italia), La Repubblica (Italia), Le Figaro (Francia), Le Monde (Francia), The Guardian (Reino Unido) y The Times (Reino Unido). Estos diarios, en cinco idiomas (árabe, francés, inglés, italiano y castellano) se escogieron por diferentes razones: por su relevancia en cada uno de los países, por recoger una perspectiva plural desde el punto de vista de la ideología (gubernamental-oficialista/oposición, derecha/izquierda...) y en función de los objetivos y recomendaciones de las organizaciones supranacionales como son la Unión por el Mediterráneo, la Política de Vecindad de la Unión Europea y la Alianza de Civilizaciones, de acuerdo a los planteamientos iniciales formulados en el proyecto de investigación.

La aportación a la muestra de cada una de las cabeceras fue distinta. Desde los 126 unidades de análisis abordadas en Al Massae a las tan solo 30 de The Guardian.

Tabla 1. Diarios analizados, procedencia e idioma utilizados

\begin{tabular}{|l|l|l|l|}
\hline Diario & Número de unidades analizadas & País & Idioma \\
\hline Al Ahram & 108 & Egipto & Árabe \\
\hline Al Dostur & 72 & Egipto & Árabe \\
\hline Al Massae & 126 & Marruecos & Árabe \\
\hline Al Thawra & 88 & Siria & Árabe \\
\hline Al Watan- Siria & 116 & Siria & Árabe \\
\hline As Sabah & 52 & Marruecos & Árabe \\
\hline El Khabar & 79 & Argelia & Árabe \\
\hline ABC & 39 & España & Castellano \\
\hline El País & 93 & España & Castellano \\
\hline
\end{tabular}




\begin{tabular}{|l|l|l|l|}
\hline El Watan & 72 & Argelia & Francés \\
\hline Il Corriere della Sera & 108 & Italia & Italiano \\
\hline La Repubblica & 66 & Italia & Italiano \\
\hline Le Figaro & 96 & Francia & Francés \\
\hline Le Monde & 69 & Francia & Francés \\
\hline The Guardian & 30 & Reino Unido & Inglés \\
\hline The Times & 40 & Reino Unido & Inglés \\
\hline
\end{tabular}

Fuente: Elaboración propia

Tabla 2. Idioma utilizado en porcentaje por las cabeceras en la muestra seleccionada.

\begin{tabular}{|l|l|}
\hline Árabe & 51,1 \\
\hline Castellano & 10,5 \\
\hline Francés & 18,9 \\
\hline Inglés & 5,6 \\
\hline Italiano & 13,9 \\
\hline
\end{tabular}

Fuente: Elaboración propia

\section{3. ¿Qué etendemos por géneros periodísticos?}

Entendemos los géneros periodísticos como las diferentes formas que se plantean para transmitir los mensajes en el ámbito del periodismo. Su evolución es constante hacia una tendencia cada vez más clara de hibridación sobre todo cuando los analizamos en los nuevos medios que tienen internet como soporte. De hecho, el concepto de género periodístico es una evolución del de género literario.

"La noción de género periodístico es naturalmente tributaria de la de género literario, pero su historia es más la de periodismo que la de la literatura. Los géneros periodísticos aparecen a medida que el periodismo evoluciona e intenta responder a las nuevas necesidades" (Gomis, 1989: 85).

Por tanto, podemos decir que los géneros periodísticos son los diferentes moldes a través de los cuales transmitimos los contenidos periodísticos.

"La elaboración de los mensajes periodísticos o, lo que es lo mismo, la forma en que deben ser redactados los mensajes para su perfecta y rápida comprensión por un público no indeterminado pero sí amplio, nos lleva al planteamiento de los géneros periodísticos" (Caminos y Armentia, 1997: 61).

Así, Muñoz asegura que "los géneros periodísticos son las diversas modalidades de creación lingüística que se caracterizan por acomodar sus estructuras a la difusión de noticias y opiniones a través de los medios de comunicación social" (Muñoz, 1994: 121). 
Por su parte, Gomis explica que "el género representa, por decirlo así, una suma de artificios estéticos a disposición del escritor e inteligibles para el lector" (Gomis, 1989: 84). Añade que:

“... facilitan el trabajo en común. Cuanto más respeten las convenciones propias del género - nacidas entre una peculiar relación entre contenido y la forma- más homogéneo resultará el trabajo de redacción y más confianza depositará el receptor en el mensaje que le llega" (1989: 98-99).

Martínez Albertos afirma que los géneros periodísticos son aquellas modalidades de la creación literaria concebidas como vehículos aptos para realizar una estricta información de actualidad (o periodismo) y que están destinados a canalizarse a través de la prensa escrita (Martínez Albertos, 1993: 264).

Además, señala que:

"los géneros periodísticos deben ser para nosotros principios de conocimientos del mensaje informativo, en su dimensión de texto literario, teniendo en cuenta que este mensaje es de alguna manera la expresión de las posibilidades humanas para lograr un cierto grado de comunicación de hechos y de ideas mediante un no desdeñable nivel de creación estética en el uso de la palabra" (Martínez Albertos, 1993: 267).

"Los géneros periodísticos se presentan como manifestaciones concretas y singulares en las que se plasma efectivamente todo ese caudal de recursos expresivos o de rasgos de redacción que es capaz de almacenar en sí un individuo determinado" (López, 1999: 103).

Hacer una clasificación de géneros periodísticos no siempre es una tarea sencilla. De hecho si se repasa la amplia bibliografía existente al respecto podemos comprobar disparidad de criterios.

Muñoz habla de distintos tipos de géneros periodísticos. Aportamos dos.

"La primera gran división genérica, ya mencionada, es la que separa los hechos (stories: exposición aséptica, transmisión, relato, constatación) de las opiniones sobre esos hechos (comments: comentarios, opiniones, valoraciones). Se establecen tradicionalmente tres niveles de información (informativo puro, interpretación y opinión (...)" (Muñoz, 1994: 123).

Además ofrece una segunda clasificación basada en cinco variables: informativo puro; ambiguo mixto de información e interpretación; interpretativo; ambiguo-mixto de interpretación y opinión; y de opinión. (Muñoz, 1994: 125).

Por su parte, Casasús y Núñez Ladevéze también estudian este ámbito y realizan esencialmente una doble aportación.

"La dimensión objetiva de los géneros nos conduce hacia la apreciación de modelos estructurales y estilísticos cuyos conjuntos prototípicos reciben diversas denominaciones (noticia o información, crónica, reportaje, artículo, editorial, crítica, etc.). Por su parte, la dimensión subjetiva se vertebra y clasifica según su contenido temático (político, económico, mundano, científico, deportivo, etc.). Los modelos de género se constituyen mediante la combinación de ambas dimensiones. Así obtene- 
mos, por ejemplo, la crónica deportiva, el reportaje político, la información científica o la crítica musical” (Casasús y Núñez Ladevéze, 1991: 87).

Además, también señalan que los diferentes géneros periodísticos se pueden establecer en función de "los grandes grupos clásicos (...) géneros informativos (...), géneros interpretativos (...), géneros argumentativos (...) y géneros instrumentales” (Casasús y Núñez Ladevéze, 1991: 88).

Pero además, Núñez Ladevéze considera que se pueden distinguir tres tipos de géneros periodísticos. "De información, de interpretación y de opinión; a los que corresponden tres tipos diferenciados de periodismo; el principalmente informativo, el interpretativo y el de opinión" (Núñez Ladevéze, 1995: 34).

Como se puede observar en estas propuestas, los autores se centran prácticamente de forma exclusiva en la prensa escrita y, de este modo, solamente hablan de cómo trasladar la información y los comentarios, los hechos y las opiniones, pero siempre haciendo referencia a los aspectos noticiables.

En función de estas características, cada uno de ellos diseña una clasificación diferente, aunque con una base común. La excepción es la que protagonizan en una de sus reflexiones Casasús y Núñez Lavédeze (1991: 87). Ambos teóricos señalan que los géneros periodísticos son la combinación tanto del formato mediante el cual es transmitido un tema (crónica, noticia, reportaje, ....), como del contenido emitido (política, deporte, economía ...). Es decir, indican que podemos encontrar la crónica deportiva, pero también la política, por poner algún ejemplo.

Compartimos con Casasús y Núñez Lavédeze esta reflexión. Sin embargo, nosotros no consideramos que los géneros se puedan definir en función de unos temas, sino que los contenidos se transmiten mediante diversas formas, es decir, a través de varios géneros. Coincidimos en que puede haber, como han ejemplarizado, crónicas deportivas, políticas,..., aunque hay que indicar que pensamos que el género es únicamente la crónica sin tener en cuenta la materia abordada.

Por tanto, y tomando como válida la propuesta formulada por Sabés y Verón (2008: 32-41) podemos establecer la existencia de géneros informativos (transmiten información), interpretativos (opinión) e híbridos (combinan la información con elementos subjetivos).

Como géneros informativos observamos la noticia, el reportaje y la entrevista. Asimismo, los principales géneros interpretativos son: editorial, artículo de fondo, comentario de actualidad, tribuna libre y cartas al director. En cuanto a los géneros híbridos destacamos la crónica, el reportaje interpretativo y la crítica. De estos últimos queremos detenernos en la crónica.

"La crónica se caracteriza por ser un texto que aborda un hecho noticioso, pero en el que se ofrece la visión personal del autor. Es decir, es la información contada desde la perspectiva de quien, pero no hay que olvidar nunca que es información lo que se aporta" (Sabés y Verón, 2008: 39). 
En la investigación que se presenta se analiza la información aparecida en la prensa relativa al espacio euromediterráneo. Por este motivo, se estudian los géneros periodísticos informativos (noticia, reportaje y entrevista), pero también se decidió incluir la crónica, que pese a ser híbrido la información prima sobre los elementos valorativos del autor.

Gráfico 1. Cuadro resumen de los principales géneros periodísticos.

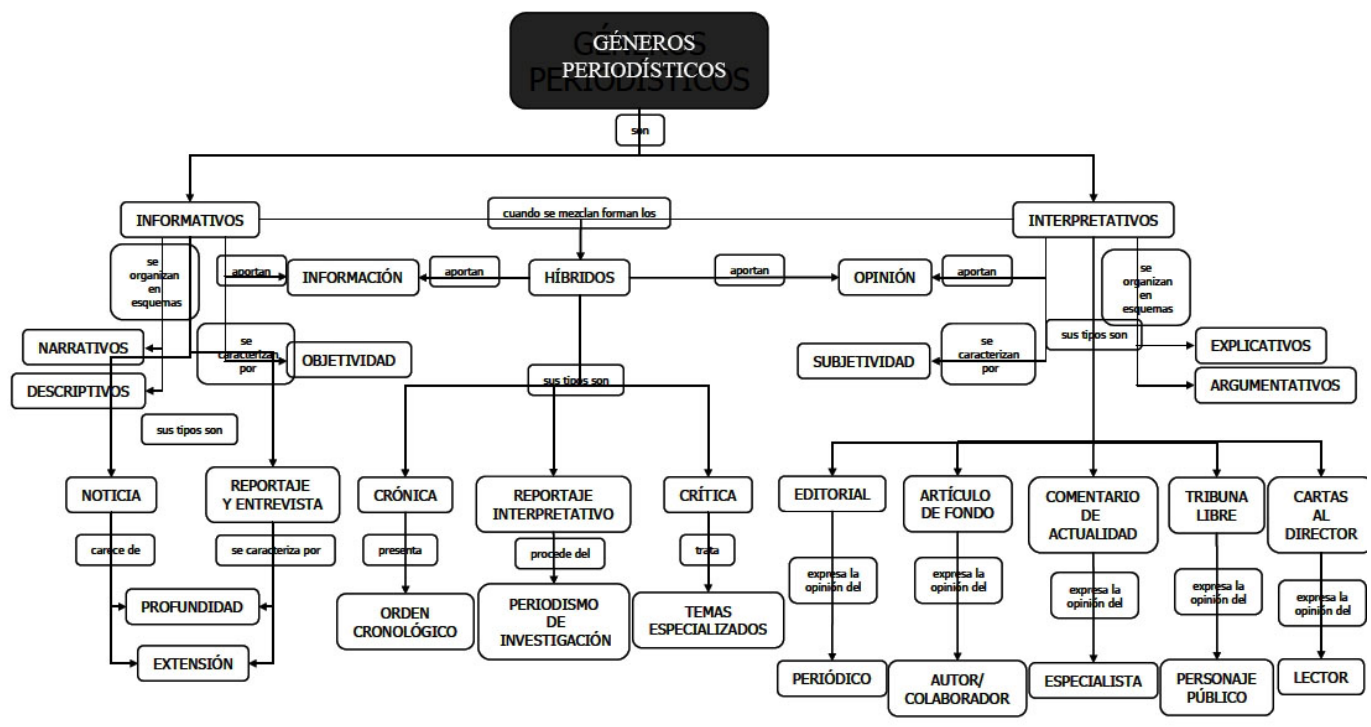

Fuente: Sabés, F. y Verón, J.J. (2008).

\section{Análisis de resultados}

Los resultados que se presentan hacen referencia a la utilización de los géneros periodísticos informativos en 16 diarios del espacio euromediterráneo durante dos semanas en las que se recogió la muestra analizada. Lo que se pretende es poner de manifiesto el tratamiento en los distintos medios de comunicación de las informaciones que hacían referencia a las categorías establecidas en el proyecto de investigación anteriormente citado.

De esta manera, hay que indicar que la participación de las distintas cabeceras en la muestra oscila entre el $1 \%$ y el $11 \%$. 


\section{Gráfico 2. Reparto de la muestra por diarios analizados.}

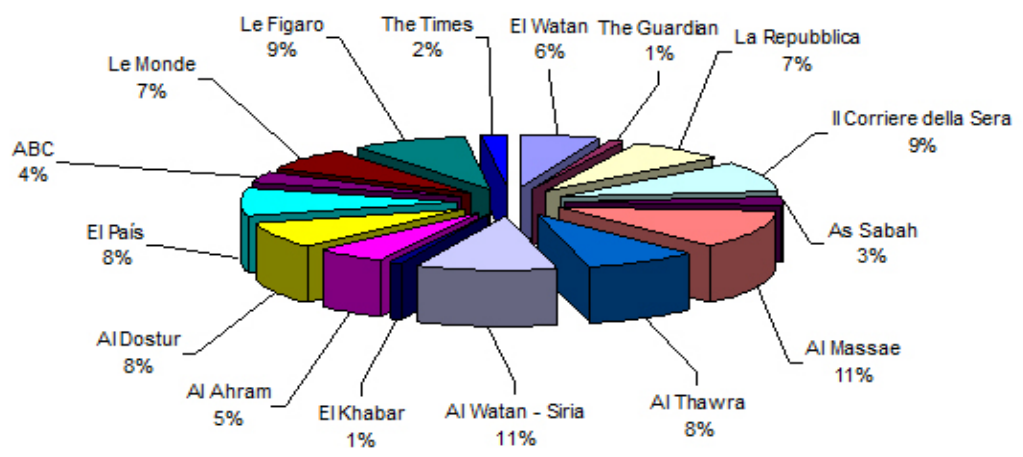

Fuente: Todos los gráficos incluidos en este artículo son de elaboración propia

Los dos medios que aportan más contenidos vinculados a la región euromediterránea y a los incluidos en la pauta de análisis son Al Watan Siria y en Al Massae (11\%), mientras que en el lado opuesto encontramos a los británicos The Guardian y The Times y también al argelí Al Khabar, los tres representando el 1\% de la muestra, aspecto que pone de manifiesto la priorización del espacio euromediterráneo en el tratamiento informativo en unos medios sobre otros.

Si estudiamos el reparto de los géneros periodísticos en las informaciones incluidas en la muestra, hay que indicar que la mayor parte son noticias, un 77\%. La presencia de crónicas alcanza al $13 \%$ de las piezas estudiadas; el 7\%, a reportajes y el 3\%, a entrevistas.

\section{Gráfico 3. Reparto de la muestra por géneros periodísticos.}

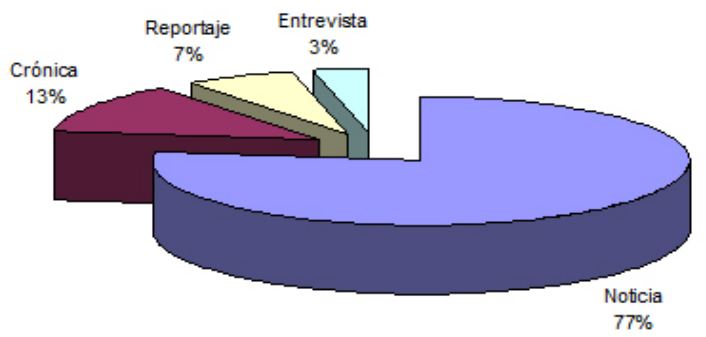

Es necesario también repasar el reparto por géneros periodísticos de las piezas estudiadas en cada uno de los medios de comunicación incluidos en la muestra.

En El Watan, el 74\% de los contenidos incluidos en el estudio se presentan bajo el formato de noticia, mientras que el $11 \%$ a través de entrevistas; el 10\%, con reportajes y únicamente el 5\% con crónicas. 
Gráfico 4. Presencia de géneros periodísticos informativos en El Watan.

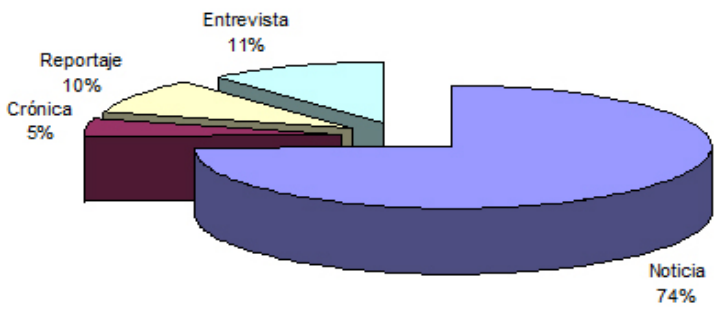

El británico The Guardian incluye en las piezas estudiadas únicamente dos géneros periodísticos informativos. Mayoritariamente presenta noticas, con un $82 \%$, frente al $18 \%$ que se ofrece mediante crónicas.

Gráfico 5. Presencia de géneros periodísticos informativos en The Guardian.

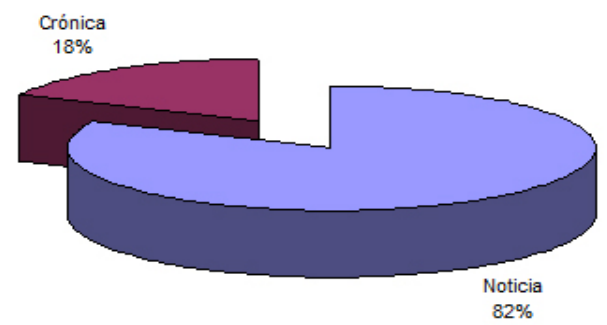

En cuanto al diario italiano La Repubblica, el género noticia alcanza el $71 \%$ de las piezas estudiadas, aunque también hallamos otros tres géneros: reportajes $(15 \%)$, crónicas (11\%) y entrevistas (3\%).

Gráfico 6. Presencia de géneros periodísticos informativos en La Repubblica.

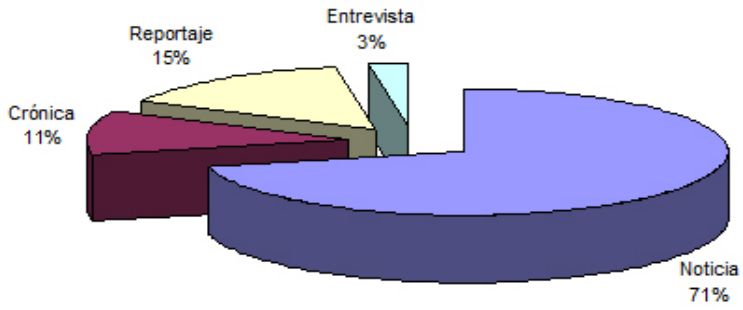

Por su parte, en el diario Il Corriere della Sera, el género noticia representa el 72\% de las publicaciones informativas que hacen referencia al espacio euromediterráneo, mientras que la crónica el 12\%; el reportaje, el 11\% y la entrevista, el 5\%. 
Gráfico 7. Presencia de géneros periodísticos informativos en Il Corriere de la Sera.

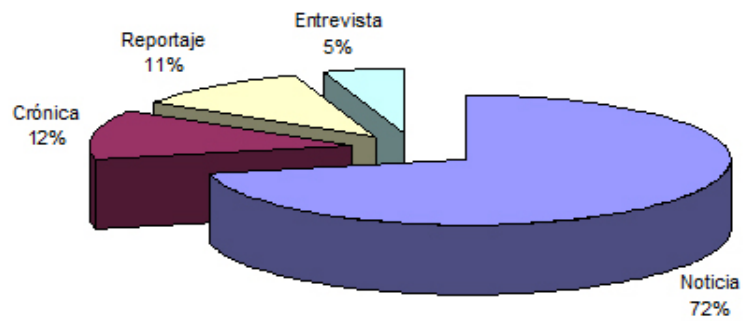

En As Sabah la variedad de géneros es mínima ya que el formato noticia suma el 93\% y la crónica, el 7\% restante.

Gráfico 8. Presencia de géneros periodísticos informativos en As Sabah.

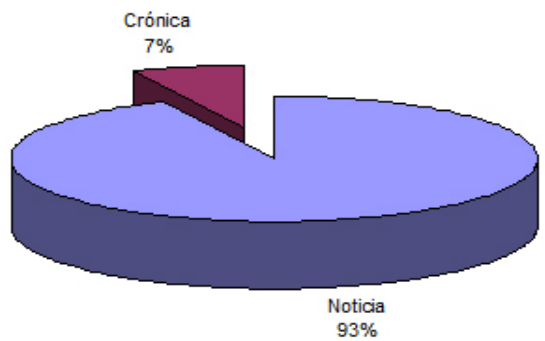

La noticia vuelve a ser el género predominante en la cabecera Al Massae, alcanzando el $83 \%$ de las piezas analizadas. Completan la presencia de la información de la región mediterránea; la entrevista el 8\%, la crónica el 7\% y el reportaje el 2\%.

Gráfico 9. Presencia de géneros periodísticos informativos en Al Massae.

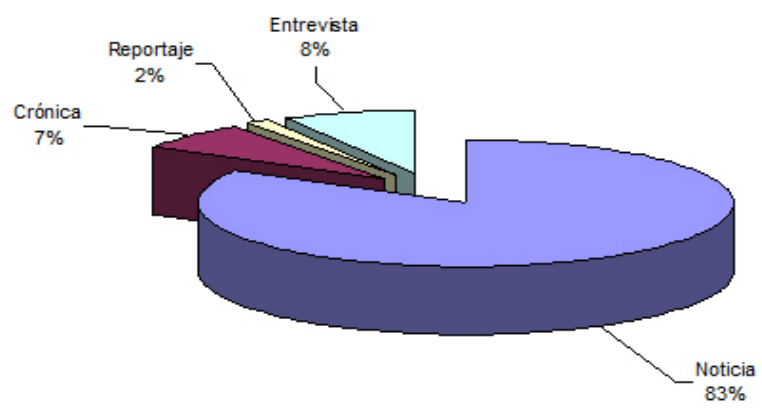

En el diario Al Thawra, las noticias representan el 91\% de las informaciones que hacen referencia al espacio euromediterráneo, mientras que el reportaje suma el 8\% y la crónica queda en un mínimo $1 \%$. 
Gráfico 10. Presencia de géneros periodísticos informativos en Al Thawra.

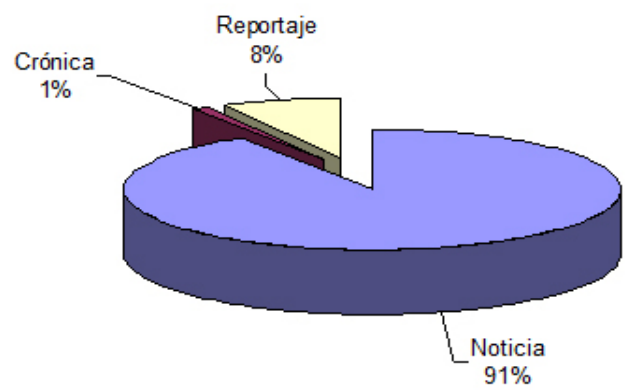

En el diario Sirio Al Watan-Siria observamos tres géneros periodísticos informativos, aunque es la noticia la que alcanza la gran mayoría de informaciones $(95 \%)$. La crónica queda reducida al 4\% y el reportaje al 1\%.

Gráfico 11. Presencia de géneros periodísticos informativos en Al Watan.

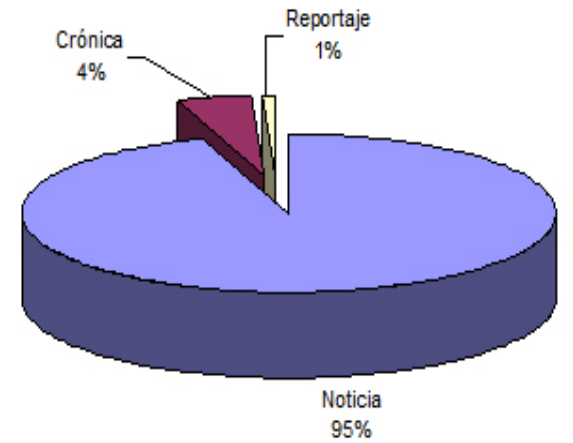

Uno de los diarios que se comportan de forma diferente a la gran mayoría de los estudiados es El Khabar, donde la noticia solamente representa el 13\%, mientras que el género informativo predominante es la crónica (87\%).

Gráfico 12. Presencia de géneros periodísticos informativos en El Khabar.

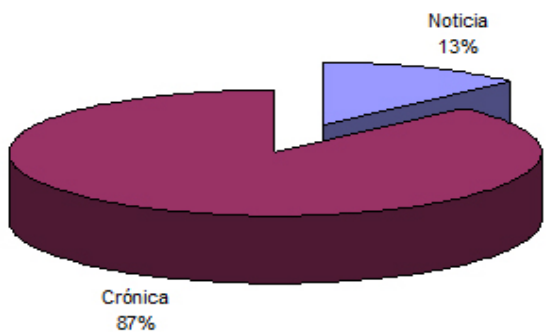


Asimismo, la cabecera Al Ahram apuesta por una extraordinaria pluralidad de géneros periodísticos informativos. Pese a que la noticia es el mayoritario con un $61 \%$, también hay que destacar el papel de la crónica (28\%). Completan los géneros el reportaje (7\%) y la entrevista (4\%).

\section{Gráfico 13. Presencia de géneros periodísticos informativos en Al Ahram.}

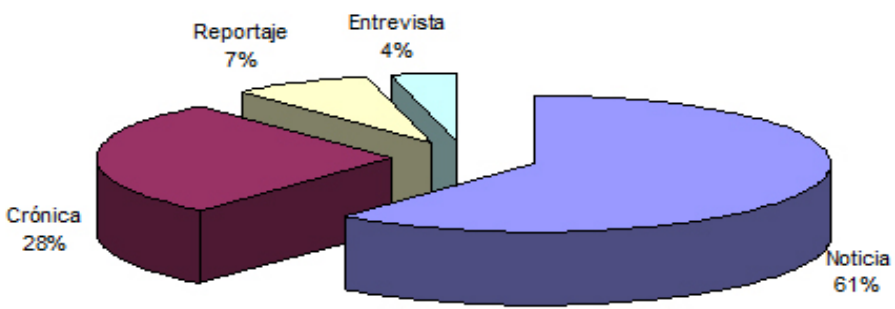

Al Dostur es la cabecera en la que la noticia no es el género predominante (18\%), siendo la crónica el mayoritario, alcanzando el $67 \%$. Completan el resto de géneros que aparecen en este diario el reportaje (13\%) y la entrevista (2\%).

Gráfico 14. Presencia de géneros periodísticos informativos en Al Dostur.

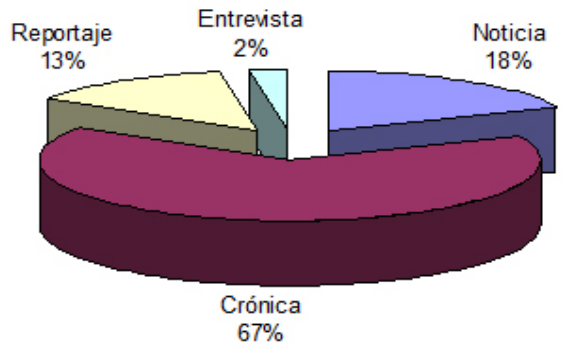

En el diario del Grupo Prisa El País, la presencia mayoritaria es de noticias al abordar asuntos vinculados con el espacio euromediterráno. De todas maneras, también se encuentran crónicas $(2 \%)$ y entrevistas $(1 \%)$, aunque ambos géneros de forma muy reducida. 
Gráfico 15. Presencia de géneros periodísticos informativos en El País.

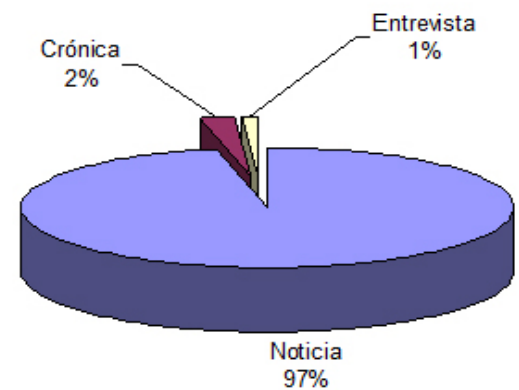

El modelo de El País prácticamente se repite en el otro diario español estudiado, $A B C$. Las noticias alcanzan el $89 \%$, seguido del reportaje (\%) y de la entrevista $(3 \%)$.

Gráfico 16. Presencia de géneros periodísticos informativos en $\mathrm{ABC}$.

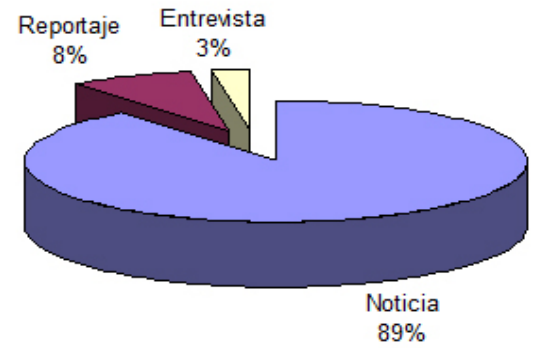

En Le Monde, el 71\% de los contenidos informativos vinculados a la materia aparecen bajo el género de noticia, mientras que el 13\% se muestran como reportajes; el $9 \%$ a través de crónicas y el $7 \%$ por medio de entrevistas.

Gráfico 17. Presencia de géneros periodísticos informativos en Le Monde.

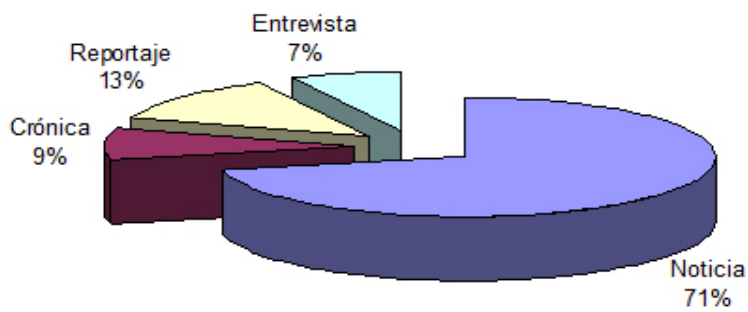

En Le Figaro, la crónica y el reportaje representan cada uno de ellos el 8\% de las informaciones relativas al espacio euromediterráneo. Por su parte el $84 \%$ de las informaciones son noticias. 


\section{Gráfico 18. Presencia de géneros periodísticos informativos en Le Figaro.}

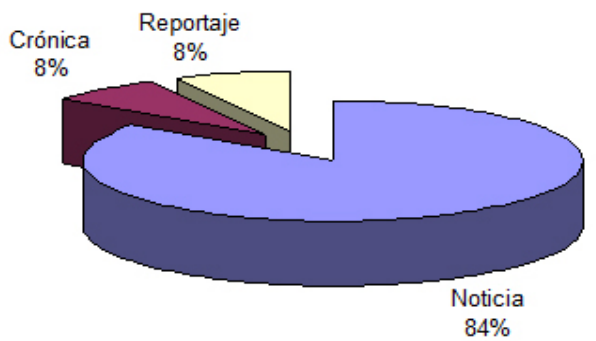

En cuanto al británico The Times, la noticia es el género que prácticamente abarca toda la presencia de contenidos vinculados al espacio euromediterráneo (95\%). Únicamente observamos un 5\% de crónicas.

Gráfico 19. Presencia de géneros periodísticos informativos en The Times.

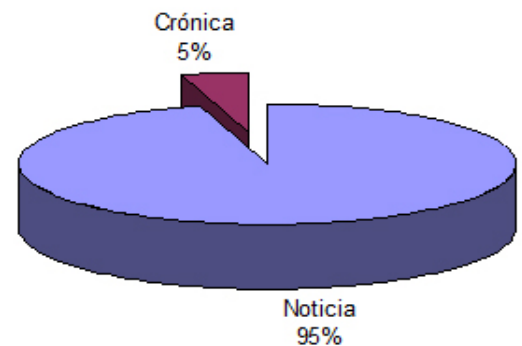

\section{Conclusiones}

El estudio de la información relativa al espacio euromediterráneo que publican los medios de comunicación es un interesante fenómeno a trabajar y en esa línea se enmarca este artículo y los proyectos de investigación anteriormente enunciados en los que entre otros los autores están implicados. La relevancia internacional de esta área es cada vez mayor y es previsible que lo continúe siendo en las próximas décadas.

El análisis de la información generada sobre este ámbito, en este caso de la prensa, pone de manifiesto esencialmente la escasa innovación que evidencian todas las publicaciones analizadas desde el punto de vista formal. Sería conteniente después del resultado de este primer análisis continuar en esta línea de investigación y poder realizar comparaciones con otros medios de comunicación que se publican en otros soportes, sobre todo en la red, sin olvidar las versiones digitales de las cabeceras analizadas.

Si retomamos el estudio planteado la escasa innovación en los géneros periodísticos se evidencia con que el $77 \%$ de las informativas relativas a este tema se publican 
bajo el formato noticia; únicamente un 13\% a través de crónicas, un $7 \%$ con reportajes y un 3\% por medio de entrevistas.

Pero si profundizamos en los medios estudiados solo dos medios presenta más crónicas que noticias: Al Dostur, $67 \%$ frente al $18 \%$, que se completa con un $13 \%$ de reportajes y un $2 \%$ de entrevistas, y El Khabar, $87 \%$ frente a $13 \%$.

El resto de cabeceras analizadas se comportan de forma contraria: El Watan (74\%), The Guardian (82\%), La Repubblica (71\%), Il Corriere de la Sera (72\%), As Sabah (93\%), Al Massae (83\%), Al Thawra (91\%), Al Watan Siria (95\%), Al Ahram (61\%), Al Dostur (67\%), El Pais (97\%), ABC (89\%), Le Monde (71\%), Le Figaro (84\%) y The Times (95\%).

Estos datos evidencian un comportamiento prácticamente común de todos los medios abordados, en los que la noticia es el género informativo que más aparece a la hora de plantear los temas relativos al área euromediterránea.

No deja de sorprender la falta de diversidad en la mayor parte de los diarios y su escasa innovación.

Por todo ello, reiteramos la necesidad de seguir estudiando desde diversas perspectivas y en diferentes medios el tratamiento y la temática de las informaciones que se publican a un lado y otro del mar Mediterráneo. Un análisis de los géneros periodísticos nos ayudará también a entender la evolución del periodismo que se produce en los principales países de la zona.

\section{Referencias bibliográficas}

BARDIN, Laurence (1986). Análisis de contenido. Madrid: AKAL.

BERELSON, Bernad (1952). Content analysis in comunication research. Clencoe: Free press.

BERGUER, PETER L. Y LUCKMAN, THOMAS (1976). La construcción social de la realidad. Buenos Aires. Amorrortu.

CAMINOS, José María; ARMENTIA, José Ignacio (1997). Principios básicos de la noticia escrita. Bilbao. Universidad del País Vasco.

CASASÚS, Josep Maria; NÚÑEZ LADEVÉZE, Luis (1991). Estilo y géneros periodisticos. Barcelona: Ariel.

GOMIS, Lorenzo (1989). Teoria del generes periodístics. Barcelona: Centre d'Investigació de la Comunicació de la Generalitat de Catalunya.

HOLSTI, OLE R. (1968): “Content analysis". En LINDZEY, G. Y ARONSON, E. The handbook of social Psychology. Vol 2. Research Methods, Addison-Wesley, Reading, Mass.

KRIPPENDORFF, Klaus (1990). Metodología del análisis de contenido. Teoría y práctica. Barcelona. Paidós Comunicación. 
LÓPEZ, Xosé (1999). Comunicación e información escrita. Redacción periodística. Santiago de Compostela. Edicions Lea.

MARTÍNEZ ALBERTOS, José Luis (1993). Curso general de redacción periodística. Lenguaje, estilos y géneros periodísticos en prensa, radio, televisión y cine. Madrid. Paraninfo.

MUÑOZ, José. (1994). Redacción periodística. Teoría y práctica. Salamanca. Librería Cervantes.

NÚÑEZ LADEVÉZE, Luis (1995). Introducción al periodismo escrito. Barcelona. Ariel.

RIZO, Marta (2009). "Sociología fenomenológica y comunicología: Sociología Fenomenológica y sus aportes a la comunicación interpersonal y mediática". En Revista Fronteiras-Estudos Midiáticos. Nún 11 (1): 25-32, janeiro/abril 2009.

SABÉS, Fernando y VERÓN, José Juan (2008). La eficacia de lo sencillo. Introducción a la práctica del Periodismo. Sevilla. Comunicación Social Ediciones y Publicaciones.

VELÁZQUEZ, Teresa (1992). Los políticos y la televisión. Aportaciones de la teoría del discurso al diálogo televisivo. Barcelona. Ariel.

VELÁZQUEZ, Teresa (2011): "Las técnicas cualitativas del análisis socio-semiótico". En: VILCHES, Lorenzo (coord.): La investigación en comunicación. Métodos y técnicas en la era digital. Barcelona: Gedisa, cap. 8: 237-265.

\section{Los autores}

Fernando Sabés Turno es doctor en Comunicación Audiovisual y Publicidad por la Universidad Autónoma de Barcelona. Es profesor titular en el departamento de Periodismo y Ciencias de la Comunicación de la Facultad de Ciencias de la Comunicación de la Universidad Autónoma de Barcelona, donde ha sido vicedecano y actualmente es vicedirector de docencia del mencionado departamento. Ha escrito diferentes libros entre los que destacan los elaborados junto a José Juan Verón Lassa: La eficacia de lo sencillo. Introducción a la práctica del periodismo (2008) y La gestión de la información en la administración local (2008). También ha escrito numerosos artículos en revistas científicas del ámbito de la comunicación y varios capítulos de libros.

Ricardo Carniel Bugs es Doctor en Comunicación y Periodismo por la Universidad Autónoma de Barcelona y periodista. Es profesor investigador postdoctoral en el departamento de Periodismo y Ciencias de la Comunicación de la Facultad de Ciencias de la Comunicación de la UAB. Es miembro del Laboratori de Prospectiva i Recerca en Comunicació, Cultura i Cooperació (LAPREC) y del Observatorio Mediterráneo de la Comunicación (OMEC) de la UAB. Ha publicado artículos y capítulos de libros sobre estructura y políticas de comunicación, con énfasis en la regulación de los medios audiovisuales. 